\title{
Introduction to the Issue on Gossiping Algorithms Design and Applications
}

A $\mathrm{N}$ inevitable consequence of the scaling of electronics is that in today's world it is hard to think of sensing problems that do not involve a multitude of networked platforms. Perhaps this explains why, in recent years, distributed consensus and gossiping algorithms have received considerable attention in many disciplines, and spurred a number of new research results on distributed signal processing.

These algorithms provide solutions to important problems in sensor networks, localization, robotics, distributed systems' calibration, and distributed compressive field sensing. Unlike data aggregation schemes that concentrate raw data to be processed in a central server, gossip algorithms are iterative and blend communications and computation as a network diffusion process. They provide simple and instructive abstractions of decentralized signal processing, involving key features of this class of problems, such as interaction among many terminals, subject to a model of the cost of communication and to connectivity constraints. The most appealing feature of gossiping algorithms is that in most of them very little information about the network beyond the immediate neighborhood is available at each node, and many methods are naturally resilient to link and node failures. The performance of gossip algorithms allow to make many novel connections to mathematical tools pertaining to graph theory, that are enriching the research in signal processing.

The objective of this special issue is to highlight some of the interesting directions this research has taken.

One prolific research direction is to cast an important class of classic inference problems and optimization, as network gossiping algorithms. Many of the papers in this special issue fall in this class and stretch the boundaries of what can be computed by network diffusion. Interestingly, these efforts provide increasing evidence that networks can exhibit emergent cognitive behavior, even if the data exchanged and computations performed are relatively simple.

For example, "Mobile Adaptive Networks" by S.-Y. Tu and A. H. Sayed, investigate the self-organization and cognitive abilities of adaptive networks to pursue a target, fusing local information via a gossip based adaptive filter. Another important instance of sensor fusion via gossiping is demonstrated in the paper "Gossip-Based Algorithm for Joint Signature Estimation and Node Calibration in Sensor Networks" by N. Ramakrishnan et al. "Convergence Rate Analysis of Distributed Gossip (Linear Parameter) Estimation: Fundamental Limits and Tradeoffs" by S. Kar and J. M. F. Moura, also includes important results on the convergence of linear estimators via network diffusion. Gossip-based estimation maximization and maximum

Digital Object Identifier 10.1109/JSTSP.2011.2160574 likelihood are the topics investigated in "Gossip Algorithms for Simultaneous Distributed Estimation and Classification in Sensor Networks" by A. Chiuso et al.. The paper "Distributed Clustering using Wireless Sensor Networks," by P. A. Forero et al. shows that unsupervised learning can be attained by network diffusion. Also in this class is "Distributed Principal Subspace Estimation in Wireless Sensor Networks" by L. Li et al. which also shows how it is possible to decentralize the well known Oja's learning rule and learn the sample covariance principal subspace via gossiping.

Papers that are focused on using gossiping to solve a wide class of important optimization problems are: "Distributed Asymptotic Minimization of Sequences of Convex Functions by a Broadcast Adaptive Subgradient Method" by R. L. G. Cavalcante et al. "Performance Evaluation of the Consensus-based Distributed Subgradient Method Under Random Communication Topologies" by I. Matei and J. S. Baras, and "Distributed Asynchronous Constrained Stochastic Optimization" by K. Srivastava and A. Nedić.

A third group of papers is focused on analyzing or improving the performance of average consensus focusing on its ability to broadcast a function of the data under different sources of distortions and constraints. This has been one of the most prolific topics of investigation, and many authors have researched the design and analysis of resilient communication protocols over wireless networks supporting average consensus gossiping. New advances are still made especially in considering compression, finite precision in the states and interactions and noise in the communication links. Others consider the effect of mobility and interference in wireless communications. Important contributions are made in this issue also on these fronts.

A notoriously difficult problem in gossiping algorithms, it to study convergence properties of gossiping algorithms with exchanges and updates of discrete values. Significant advances on this topic are made in the paper "The Distributed Multiple Voting Problem" by F. Bénézit et al. .

In "Efficient Decentralized Approximation via Selective Gossip" by D. Üstebay et al., instead, the authors examine a specific instance of the algorithm that works of vectors, which is useful for compression and distributed transform coding. The paper "A Token Based Approach to Distributed Computation in Sensor Networks" by V. Saligrama and M. Alanyali analyzes also a different form of selectivity in the interaction, based on a token that gives permission to specific agents to exchange and update their states. The issue of precision is revisited in "Non-Asymptotic Analysis of an Optimal Algorithm for Network-Constrained Averaging With Noisy Links" N. Noorshams and M. J. Wainwright. 
"Distributed Averaging in Dynamic Networks" by S. Rajagopalan and D. Shah gives new insights on what are the effects on gossip based computations when there are changes in the network topology in terms of communication links, or changes in the values of numbers present at nodes, with nodes joining or leaving.

The papers "Convergence Speed of the Consensus Algorithm with Interference and Sparse Long-Range Connectivity," by S. Vanka, M. Haenggi, and V. Gupta and "Broadcast Gossip Averaging: Interference and Unbiasedness in Large Abelian Cayley Networks," by F. Fagnani and P. Frasca improve our understanding of the performance of consensus protocols over wireless networks plagued by interference and packet collisions.

Interestingly, "Local Interference Can Accelerate Gossip Algorithms" by B. Nazer et al. shows that interference can be transformed from foe to friend in average consensus gossiping, if one adopts appropriate channel codes.

We thank the many researchers that have contributed to this issue. The creativity and sophistication shown in their papers attests that signal processing has fully embraced network science and will contribute for many years to come novel solution to process distributed data in scalable and resilient fashions.

AnNa ScAgLione, Lead Guest Editor

University of California

Davis, CA 95616 USA

MARK COATES, Guest Editor

McGill University

Montreal, QC H3A 2T5, Canada

MichaEl GASTPAR, Guest Editor

École Polytechnique Fédérale de Lausanne

1015 Lausanne, Switzerland

JoHN TsITSIKLIS, Guest Editor

Massachusetts Institute of Technology

Cambridge, MA 02139 USA

MARTIN VETTERLI, Guest Editor

École Polytechnique Fédérale de Lausanne

1015 Lausanne, Switzerland

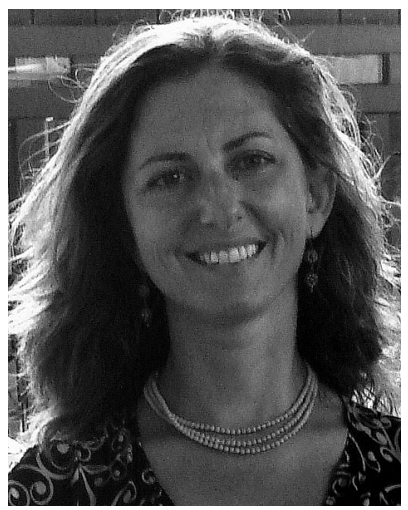

Anna Scaglione (M'99-SM'09-F'11) received the Laurea degree (M.Sc.) in 1995 and the Ph.D. degree in 1999 from the University of Rome, La Sapienza.

She is currently a Professor in Electrical and Computer Engineering at the University of California, Davis, where she joined the faculty in July 2008. She was previously at Cornell University, Ithaca, NY, starting in 2001 and received tenure in 2006; prior to this she was an Assistant Professor at the University of New Mexico in 2000 to 2001. Her expertise is in the broad area of signal processing for communication systems. Her current research focuses on cooperative wireless networks and sensors systems.

Prof. Scaglione served as an Associate Editor for the IEEE TRANSACTIONS ON WIRELESS COMMUNiCATIONS from 2002 to 2005, and from 2008 to 2011 on the Editorial Board of the IEEE Transactions on Signal PROCESSING. She has been a member of the Signal Processing for Communication Committee from 2004 to 2010. She was general chair of the workshop SPAWC 2005 and keynote speaker in SPAWC 2008. She is the first author of the paper that received the 2000 IEEE Signal Processing Transactions Best Paper Award; she has also received the NSF Career Award in 2002 and she is co-recipient of the Ellersick Best Paper Award (MILCOM 2005).

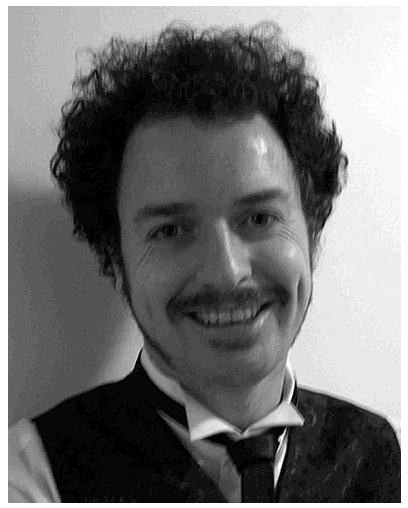

Mark J. Coates (M'99-SM'07) received the B.E. degree (first class honors) in computer systems engineering from the University of Adelaide, Adelaide, Australia, in 1995, and the Ph.D. degree in information engineering from the University of Cambridge, Cambridge, U.K., in 1999.

He joined McGill University, Montreal, QC, Canada, in 2002, where he is currently an Associate Professor. He was awarded the Texas Instruments Postdoctoral Fellowship in 1999 and was a Research Associate and Lecturer at Rice University, Houston, TX, from 1999 to 2001. His research interests include communication and sensor networks, statistical signal processing, causal analysis, and Bayesian and Monte Carlo inference. 


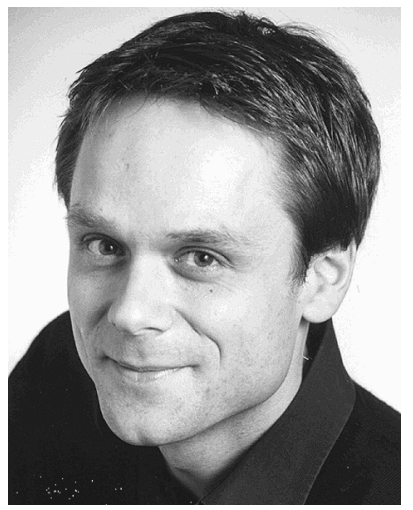

Michael Gastpar (S'99-M'04) studied engineering and philosophy in Zürich, Edinburgh, Lausanne, and Urbana-Champaign, IL, USA. He received the Dipl.El.-Ing. degree from ETH Zürich, Zürich, Switzerland, in 1997, the M.S. degree from the University of Illinois at Urbana-Champaign in 1999, and the Dr. ès sc. from Ecole Polytechnique Fédérale, Lausanne, Switzerland, in 2002.

$\mathrm{He}$ is currently a Professor at EPFL, Lausanne, Switzerland, and an Associate Professor at the University of California, Berkeley, in the Department of Electrical Engineering and Computer Sciences and in the Helen Wills Neuroscience Institute. He also holds a faculty position at Delft University of Technology, The Netherlands, and he spent time as a Researcher at Bell Laboratories, Lucent Technologies, Murray Hill, NJ. His research interests are in network information theory and related coding and signal processing techniques, with applications to sensor networks and neuroscience.

Prof. Gastpar won the 2002 EPFL Best Thesis Award, an NSF CAREER Award in 2004, an Okawa Foundation Research Grant in 2008, and an ERC Starting Grant in 2010. He is an Information Theory Society Distinguished Lecturer (2009-2011). He is currently an Associate Editor for Shannon Theory for the IEEE TRANSACTIONS ON INFORMATION THEORY, and he served as Technical Program Committee Co-Chair for the 2010 International Symposium on Information Theory, Austin, TX.

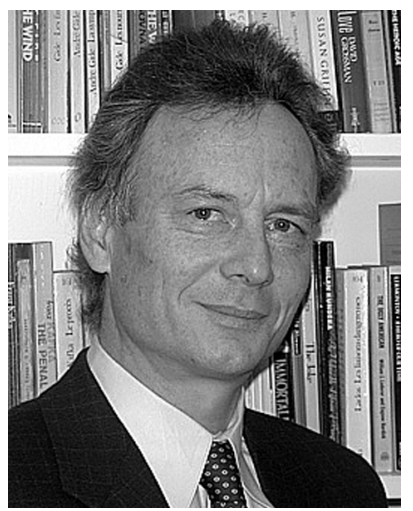

John N. Tsitsiklis (S'80-M'83-SM'97-F'99) received the B.S. degree in mathematics, and the B.S., M.S., and Ph.D. degrees in electrical engineering, all from the Massachusetts Institute of Technology (MIT), Cambridge, MA, in 1980, 1981, and 1984, respectively. During the academic year 1983-1984, he was an acting Assistant Professor of electrical engineering at Stanford University, Stanford, CA.

Since 1984, he has been with the Department of Electrical Engineering and Computer Science, MIT, where he is currently a Clarence J. Lebel Professor of Electrical Engineering. He has served as acting co-director of the Laboratory for Information and Decision Systems (Spring 1996 and 1997), and as a co-director of the Operations Research Center (2002-2005). He has also served in the National Council on Research and Technology in Greece (2005-2007). He has been a visitor with the Department of Electrical Engineering and Computer Science, University of California at Berkeley, and the Institute for Computer Science, Iraklion, Greece. His research interests are in the fields of systems, optimization, control, and operations research. He has coauthored more than 110 journal papers in these areas. He is also a coinventor in seven awarded U.S. patents. He is a coauthor with D. Bertsekas of Parallel and Distributed Computation: Numerical Methods (Athena Scientific, 1989), with D. Bertsekas Neuro-Dynamic Programming (Athena Scientific, 1996), with D. BertsimasIntroduction to Linear Optimization (Athena Scientific, 1997), and with D. Bertsekas Introduction to Probability (Athena Scientific, 2002).

He was a recipient of an IBM Faculty Development Award (1983), an NSF Presidential Young Investigator Award (1986), an Outstanding Paper Award by the IEEE Control Systems Society (1986), the MIT Edgerton Faculty Achievement Award (1989), the Bodossaki Foundation Prize (1995), and the INFORMS Computer Science Technical Section prize (1997). He is a Fellow of INFORMS (2007). In 2007, he was elected to the National Academy of Engineering. In 2008, he was conferred the title of Doctor honoris causa, from the Universite Catholique de Louvain (Belgium).

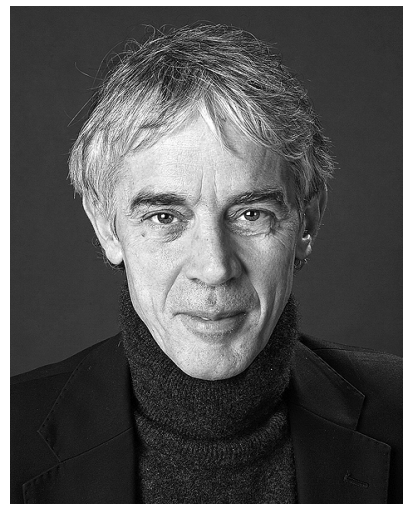

Martin Vetterli (S'86-M'86-SM'90-F'95) received the Dipl. El.-Ing. degree from ETH Zurich (ETHZ), Zurich, Switzerland, in 1981, the M.S. degree from Stanford University, Stanford, CA, in 1982, and the Doctoratés Sciences degree from EPF Lausanne (EPFL), Lausanne, Switzerland, in 1986.

He was a Research Assistant at Stanford and EPFL, and has worked for Siemens and AT\&T Bell Laboratories. In 1986, he joined Columbia University, NY, where he was last an Associate Professor of Electrical Engineering and Co-Director of the Image and Advanced Television Laboratory. In 1993, he joined the University of California at Berkeley, where he was a Professor in the Department of Electrical Engineering and Computer Sciences until 1997, and now holds an Adjunct Professor position. Since 1995, he is a Professor of Communication Systems at EPF Lausanne, Switzerland, where he chaired the Communications Systems Division (1996/1997), and heads the Audiovisual Communications Laboratory. From 2001 to 2004, he directed the National Competence Center in Research on mobile information and communication systems. He has also been a Vice-President for International Affairs at EPFL since October 2004. He has held visiting positions at ETHZ (1990) and Stanford (1998). He is the co-author, with J. Kovacevic, of the book Wavelets and Subband Coding (Prentice-Hall, 1995). He has 
published about 85 journal papers on a variety of topics in signal/image processing and communications and holds 7 patents. His research interests include sampling, wavelets, multirate signal processing, computational complexity, signal processing for communications, digital video processing, and joint source/channel coding.

Dr. Vetterli is a member of SIAM, and was the Area Editor for Speech, Image, Video, and Signal Processing of the IEEE TRANSACTIONS ON COMMUNICATIONS. He is also on the editorial boards of Annals of Telecommunications, Applied, and Computational Harmonic Analysis and The Journal of Fourier Analysis and Application. He received the Best Paper Award of EURASIP in 1984 for his paper on multidimensional subband coding, the Research Prize of the Brown Bovery Corporation (Switzerland) in 1986 for his doctoral thesis, the IEEE Signal Processing Society's Senior Awards in 1991 and in 1996 (for papers with D. LeGall and K. Ramchandran, respectively). He won the Swiss National Latsis Prize in 1996, the SPIE Presidential award in 1999, and the IEEE Signal Processing Technical Achievement Award in 2001. He was a member of the Swiss Council on Science and Technology until December 2003. He was a plenary speaker at various conferences (e.g., 1992 IEEE ICASSP). 